\title{
Can starch-based diet or sugar water put an end to the COVID-19 pandemic?
}

Jess Lan Ouyang ${ }^{1}$, Min $\mathrm{Fu}^{2}$, Jingli Peng ${ }^{1}$, Man Tang ${ }^{1}$, Qiuyun Liu ${ }^{1, *}$

${ }^{1}$ School of Life Sciences, Sun Yat-Sen University, Guangzhou 510275, China.

${ }^{2}$ Deyang Health Committee, Sichuan, China

*Correspondence author: Qiuyun Liu, School of Life Sciences, Sun Yat-sen University, Guangzhou 510275, China.

E-mail address: 1sslqy@ mail.sysu.edu.cn (Q. Liu) 


\begin{abstract}
High valine plus glycine content is a feature of the proteins in SARS-CoV-2 and SARS viruses, and it causes the generation of aggregates between proteins and insoluble calcium salts via secondary chemical bonding. Starch-based diet or sugar water with adequate vitamins can go for many days without the generation of essential amino acids such as valine, creating bottlenecks in virion production in human body. Despite its potential carcinogenicity, modest lysine supplement can be favorable as lysine rich proteins gather chloride and solubilize stressful, insoluble and stiff calcium oxalate and calcium phosphate.
\end{abstract}


The lungs of the autopsied COVID-19 patients are sticky and rigid, most likely caused by insoluble and stiff calcium salts (1-9). Organic acids tend to form rigid divalent salts. Stiff salts are most pronounced with calcium oxalate and calcium phosphate. Calorie restriction can reduce the intake of all amino acids, and minimize the production of oxalate via energy metabolism, particularly in winter when energy metabolism is in an overdrive.

\section{Conflict of interest}

None declared.

\section{REFERENCES}

1. Wan Y, Yan S, Zhang Y, An S, Yang K, Xu H, Gan T, et al. The Pneumonia outbreak: High isoleucine and high valine plus glycine contents are features of the proteins of COVID-19 virus. Preprints. 2020. doi: 10.20944/preprints202002.0289.v5

2. Lin Y, Liao L, Lam C, Ouyang J.L, Fu M, Tang M, Liu, Q. The integration of lysine supplement and weak organic acids may hold the key to a longer lifespan. OSF Preprints. 2021. osf.io/r8wby.

3. Yan S, Zhang Y, Liu Q. Why COVID-19 virus is so deadly to cancer patients? Eur J Cancer Prev. 2020;29(4):365.

4. Lam C, Lin Y, Liao L, Tang M, Fu M, Liu Q. Simultaneous fasting may contain SARS-CoV-2 infection. OSF Preprints. 2021. osf.io/zn9rg. https://osf.io/zn9rg

5. Lam C, Lin Y, Liao L, Tang M, Fu M, Liu Q. Simultaneous Implementation of Food Regimens May Contain Pandemics. OSF Preprints. 2021. https://osf.io/u3rcb

6. Lin Y, Liao L, Lam C, Ouyang J.L, Fu M, Tang M, Liu Q. Starch-based diet, plant-based diet or fasting may be beneficial to individuals suffering from sequelae of SARS-CoV-2 infection. OSF Preprints. 2021. https://osf.io/svbfy/

7. An S, Li X, Tang M, et al. The role of acetate in the antagonization of oxalate - A potential causative molecule for heart disease and cancer death. Nat Prod Commun. 2020;15(3):1-4.

8. Zhang X, Zhang J, Wang Y, Wang M, Tang M, Lin Y, Liu Q. Epigenetic Modifications and Neurodegenerative Disorders: A Biochemical Perspective. ACS Chem Neurosci. 2022;13(2):177-184.

9. Gan T, Fu M, Wu J, Wen L, Liu Q. How to design carbohydrate diet regimens for heart disease patients. Eur J Prev Cardiol. 2018; 25(9): 979-80. https://doi.org/10.1177/2047487318766602 